\title{
A Single-Crystal-Silicon Bulk-Acoustic-Mode Microresonator Oscillator
}

\author{
Joshua E.-Y. Lee, Student Member, IEEE, Behraad Bahreyni, Member, IEEE, \\ Yong Zhu, and Ashwin A. Seshia, Member, IEEE
}

\begin{abstract}
A timing reference incorporating a single-crystalsilicon micromechanical resonator with a quality factor of larger than one million and a resonant frequency of $2.18 \mathrm{MHz}$ is demonstrated. The resonator is excited in the square extensional bulk acoustic mode at $\mathbf{4}$ mtorr, and it has been fabricated in a foundry SOI MEMS process. The silicon microresonator is adapted as a timing element for a precision oscillator with a measured shortterm Allan deviation of $0.6 \mathrm{ppb}$.
\end{abstract}

Index Terms-Allan deviation, bulk acoustic resonators, high quality factor, MEMS oscillator, single-crystal silicon (SCS).

\section{INTRODUCTION}

$\mathbf{S}$ ILICON MICRO- and nanoelectromechanical resonators have been the focus of much interest in recent years, owing to their potential for a range of sensor and signal processing applications. As a material, single-crystal silicon (SCS) has been well known for its superior mechanical properties [1]. It is also known that resonators made from SCS will have lower internal losses compared with those made from polysilicon, potentially leading to higher quality factors for these devices and better performance for the oscillator designed around the resonator [2]. This, together with the possibility for integration with CMOS, batch manufacturability, and scalability have made silicon-based resonators as attractive alternatives to quartz crystals for timing applications [3], as well as realizing resonant sensors [4]. A wide variety of structural topologies have been investigated, including both flexural mode [5] and bulk acoustic mode devices [6]. Bulk-mode resonators generally have higher quality factors compared with their flexural mode counterparts [7]. In sensor and timing applications, the micromechanical resonator is typically embedded in the feedback loop of an amplifier to implement an oscillator, whose frequency stability strongly depends on the quality factor $(Q)$ of the embedded resonator.

Recently, a $Q$ of 1.6 million was demonstrated at $6 \mathrm{MHz}$ based on a square plate resonator excited in the isochoric Lame mode and operating in vacuum [8]. This letter reports a 2.18-MHz square extensional (SE) mode [9] resonator with a measured $Q$ of 1.16 million at 4 mtorr, which has been embedded in the feedback loop of a nonlinear circuit to realize a

Manuscript received March 18, 2008. This work was supported in part by the U.S. Army Soldier Systems Center and in part by the EU Framework 6 project NanoTIMER. The review of this letter was arranged by Editor Y. Taur.

The authors are with the Department of Engineering, University of Cambridge, CB2 1PZ Cambridge, U.K. (e-mail: aas41@ cam.ac.uk).

Color versions of one or more of the figures in this letter are available online at http://ieeexplore.ieee.org.

Digital Object Identifier 10.1109/LED.2008.2000643

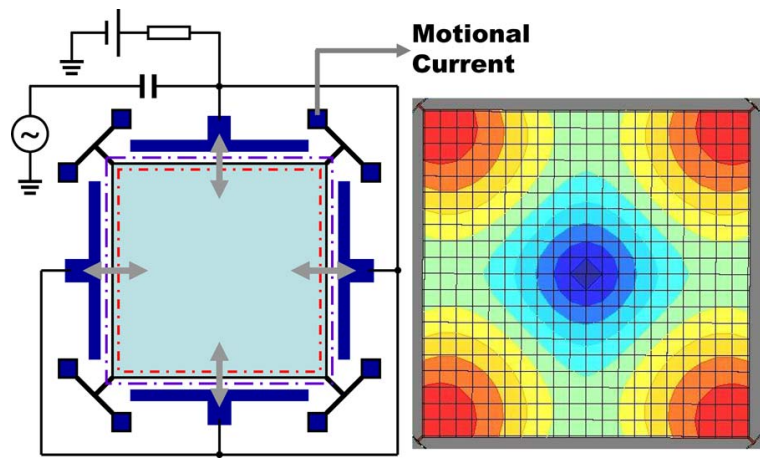

Fig. 1. Schematic illustration of the SE mode. On the right is a graded contour plot of SE mode simulated by using INTELLISUITE, showing maximum displacement at the corners.

MEMS resonator-based oscillator. We have previously reported on devices with the same topology for applications in multiarrayed parallel mass detection [10]. In this letter, we report Allan deviation measurements of a square wave oscillator, incorporating the high $Q$ SE mode resonator, and compare it to that of a commercial quartz crystal waveform generator. Details on the oscillator circuit topology are also elucidated.

\section{Resonator Model AND Design}

The SE mode may be described as a square plate that is contracting and extending symmetrically on all four sides, as shown in Fig. 1. The square plate is excited into the SE mode through lateral capacitive gap drive electrodes on each side of the structure. The motion of the structure is monitored through a motional sense current that results from the changes in the capacitance across the modulated transduction gaps. The mechanical resonant frequency of the SE mode of such structure of side length $L$ can be approximately written as [9]

$$
f_{0}=\sqrt{E / 4 \rho L^{2}}
$$

where $\rho$ is the mass density of silicon $\left(2330 \mathrm{~kg} / \mathrm{m}^{3}\right)$, and $E$ denotes the biaxial Young's modulus $(180 \mathrm{GPa})$. For a resonator of side length $L=2 \mathrm{~mm}$, (1) predicts a resonant frequency of about $2.18 \mathrm{MHz}$. This agrees well with the solutions obtained from finite-element analysis carried out in INTELLISUITE, as well as the experimental measurements.

The resonator was fabricated in a commercial foundry SOI MEMS process that is available through MEMSCAP. The capacitive transduction gap is designed to be $3 \mu \mathrm{m}$, which 


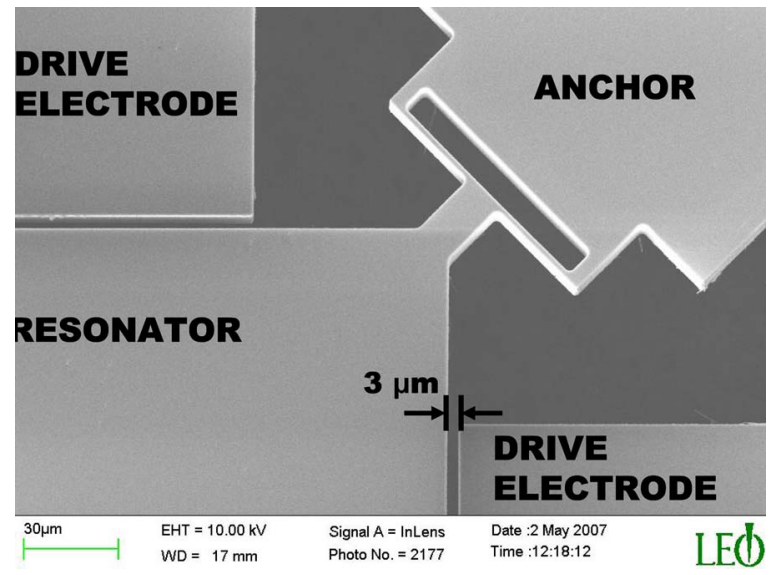

Fig. 2. SEM of the resonator fabricated in the foundry SOI MEMS process by MEMSCAP. Shown in the figure are a section of the square plate resonator, drive electrodes, and T-anchors.

is slightly wider than the minimum gap size $(2 \mu \mathrm{m})$ allowed by the foundry process. In the MEMSCAP SOI process, the movable structures are released by trench etching through the substrate, using a backside deep RIE, followed by removal of the buried oxide by a wet etch. The removal of the underlying substrate greatly reduces parasitics between the device layer and the substrate, which is beneficial for sensing small motional currents at moderately high frequencies (megahertz range). Fig. 2 shows an SEM of a corner of a fabricated resonator.

The resonator was characterized by using an Agilent 4396B network analyzer and a transimpedance $(10 \mathrm{k} \Omega)$ preamplifier to obtain its open loop transmission characteristics. A singleended-to-differential drive amplifier with a tuned compensating capacitor was used to cancel a significant fraction of the capacitive parasitic feedthrough contribution. The resonator was driven with a $-40-\mathrm{dBm}$ power source and a $60-\mathrm{V}$ dc bias for measurements reported here. The measurements were conducted in a purpose-built vacuum chamber at a pressure of 4 mtorr.

As can be seen in Fig. 3, no antiresonance is observed in the measured magnitude and phase of the frequency response, demonstrating the cancellation of capacitive parasitics. The $Q$ of the resonator obtained from these measurements is found to be $1.16 \times 10^{6}$.

\section{OSCILLATOR IMPLEMENTATION}

The SE mode resonator was embedded in the feedback loop of an amplifier to realize an oscillator. Fig. 4 shows a block diagram of the oscillator circuit. The first stage is a transimpedance amplifier to convert the resonator motional current to a voltage. This block is followed by a bandpass filter which removes unwanted oscillator modes which are likely to occur due to the large loop gains. The filtered signal is fed to a comparator. As a hard voltage limiter, the comparator allows for excellent control over the actuation signal amplitude (with the aid of a voltage divider if necessary) and effectively removes the amplitude noise of the loop signal. The comparator output is applied to the input of a single-ended-to-differential drive

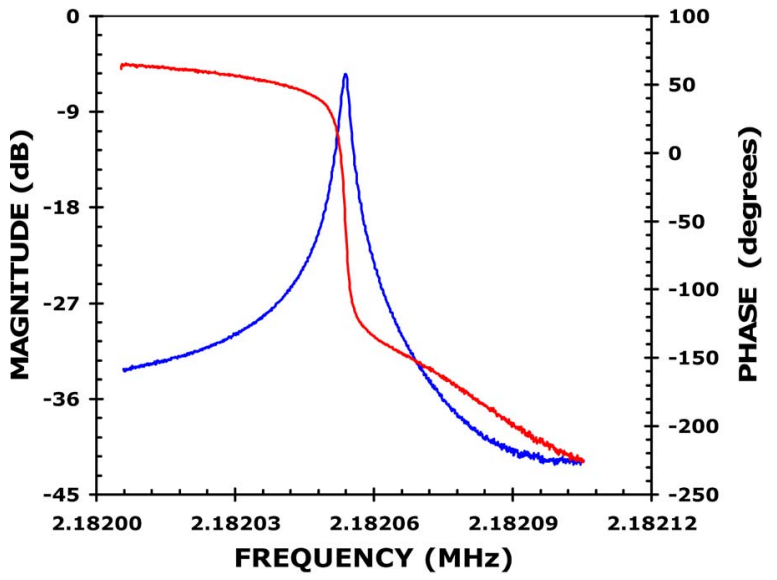

Fig. 3. Measurement of the transmission characteristic curves for the SE mode resonator at 4 mtorr (magnitude and phase), showing no antiresonance and a full $180^{\circ}$ shift at resonance.

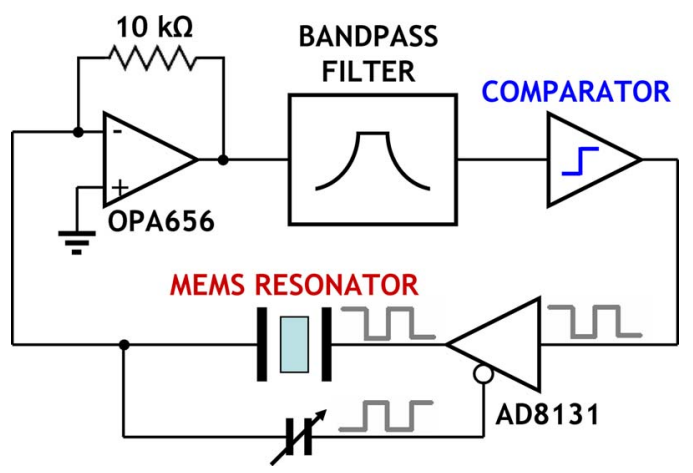

Fig. 4. Block diagram of the oscillator circuit with the SE mode resonator shown, which is embedded in the feedback loop of the circuit. The single-ended-to-differential driving amplifier reduces the effects of capacitive feedthrough.

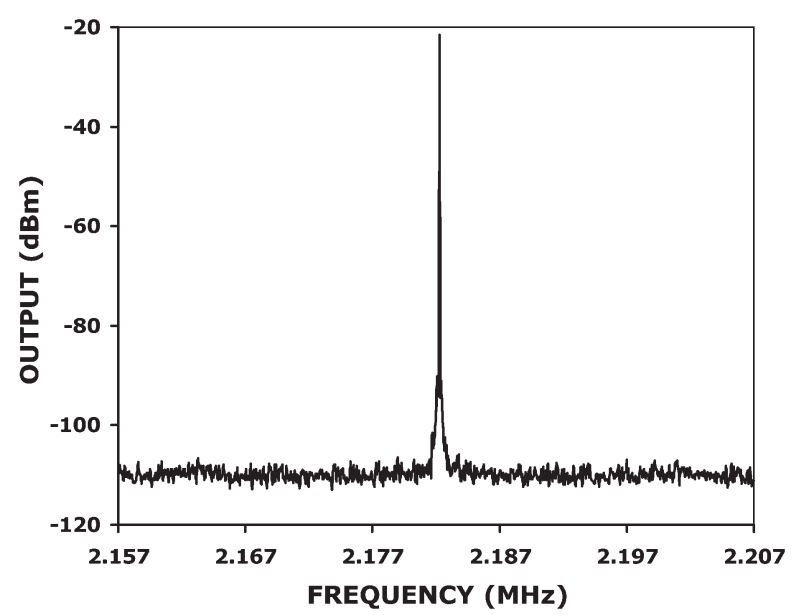

Fig. 5. Output of the MEMS oscillator showing a primary frequency component at $2.182 \mathrm{MHz}$.

amplifier, which produces two complementary binary signals at its output. These two signals are applied to the resonator and the compensation capacitor, whose output currents are added to each other at the input of the transimpedance amplifier. 


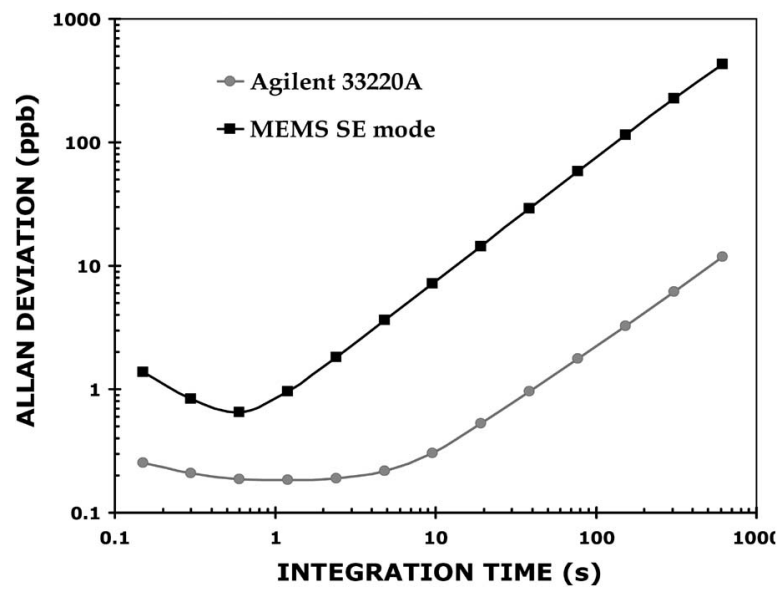

Fig. 6. Allan deviation measurements for the silicon-resonator-based oscillator and that of a voltage-controlled crystal oscillator (Agilent 33220A).

The output spectrum of the MEMS oscillator is shown in Fig. 5. Successive samples of the oscillator output frequency were obtained by using a frequency counter (Agilent 53132A) at fixed time intervals of $0.15 \mathrm{~s}$, from which the Allan deviation of the oscillator output frequency was calculated. The measured Allan deviation of the MEMS oscillator is shown in Fig. 6, which also shows the Allan deviation of a commercial voltagecontrolled crystal oscillator (Agilent 33220A) as a reference to compare the frequency stability of both oscillators.

Fig. 6 shows that the short term stability of the SE mode MEMS oscillator is comparable to the crystal oscillator, indicating that the close-to-carrier phase noise performance of the MEMS oscillator is likely to be comparable as well. The best Allan deviation for the MEMS oscillator is observed to be $0.6 \mathrm{ppb}$ for an integration time of $0.6 \mathrm{~s}$. The data points for the calculation of the Allan deviation of the signal were collected over 40 min without using any temperature compensation for the oscillator circuit, during which a steady incremental drift in frequency of up to $2 \mathrm{ppm}$ was observed. The increase in the Allan deviation of the MEMS oscillator for long integration times stems mainly from the variations in environmental temperature and pressure fluctuations inside the vacuum chamber. The slow variations in temperature affect the performance of the resonator, as well as the electronics. In addition, the pressure inside the vacuum chamber containing the MEMS resonator fluctuated, as the measurements were conducted while the chamber was continuously pumped down with a roughing pump and was stable within $+/-0.1$ mtorr of around 3.5 mtorr. The theoretical minimum for frequency fluctuations in the limit of thermomechanical noise for this device is estimated to be on the order of $10^{-12}$.

\section{CONCLUSiON}

This letter has demonstrated an SCS 2.18-MHz bulk-mode resonator with a high $Q$ of approximately 1.16 million, which is fabricated in a commercial foundry SOI MEMS process. The MEMS resonator was embedded in a feedback loop of a nonlinear circuit to realize an oscillator, which had a short term ( $\tau=0.6 \mathrm{~s})$ Allan deviation of $0.6 \mathrm{ppb}$, which is comparable with that of a commercial quartz crystal oscillator. Further improvements in frequency stability could be achieved by incorporating methods for temperature compensation and by vacuum packaging the resonator to reduce ambient pressure fluctuations [11].

\section{ACKNOWLEDGMENT}

The authors would like to thank I. Haneef for wire bonding the devices presented in this letter.

\section{REFERENCES}

[1] K. E. Petersen, "Silicon as a mechanical material," Proc. IEEE, vol. 70 , no. 5, pp. 420-457, May 1982.

[2] V. T. Srikar and S. D. Senturia, "Thermoelastic damping in fine-grained polysilicon flexural beam resonators," J. Microelectromech. Syst., vol. 11, no. 5, pp. 499-504, Oct. 2002.

[3] M. A. Hopcroft, H. K. Lee, B. Kim, R. Melamud, S. Chandorkar, M. Agarwal, C. M. Jha, J. Salvia, G. Bahl, H. Mehta, and T. W. Kenny, "A high-stability MEMS frequency reference," in Proc. 14th Int. Conf. Solid State Sens., Actuators, Microsyst. (Transducers), Lyon, France, Jun. 2007, pp. 1307-1310.

[4] G. Stemme, "Resonant silicon sensors," J. Micromech. Microeng., vol. 1, no. 2, pp. 113-125, Jun. 1991.

[5] S. Pourkamali, A. Hashimura, R. Abdolvand, G. K. Ho, A. Erbil, and F. Ayazi, "High- $Q$ single-crystal silicon HARPSS capacitive beam resonators with self-aligned sub-100-nm transduction gaps," J. Microelectromech. Syst., vol. 12, no. 4, pp. 487-496, Aug. 2003.

[6] Z. Hao, S. Pourkamali, and F. Ayazi, "VHF single-crystal silicon elliptic bulk-mode capacitive disk resonators-Part I: Design and modelling," $J$. Microelectromech. Syst., vol. 13, no. 6, pp. 1043-1053, Dec. 2004.

[7] T. Mattila, J. Kiihamaki, T. Lamminmaki, O. Jaakola, P. Rantakari, A. Oja, H. Seppa, H. Kattelus, and I. Tittonen, "A 12 MHz micromechanical bulk acoustic mode oscillator," Sens. Actuators A, Phys., vol. 101, no. 1, pp. 1-9, Sep. 2002.

[8] L. Khine, M. Palaniapan, and W. K. Wong, "6 MHz bulk-mode resonator with $Q$ values exceeding one million," in Proc. 14th Int. Conf. SolidState Sens., Actuators, Microsyst. (Transducers), Lyon, France, Jun. 2007, pp. 2445-2448.

[9] V. Kaajakari, T. Mattila, A. Oja, J. Kiihamaki, and H. Seppa, "Square extensional mode single-crystal silicon micromechanical resonator for lowphase noise oscillator applications," IEEE Electron Device Lett., vol. 25, no. 4, pp. 173-175, Apr. 2004.

[10] J. E. Y. Lee, B. Bahreyni, Y. Zhu, and A. A. Seshia, "Ultra-sensitive mass balance based on a bulk acoustic mode single-crystal resonator," App. Phys. Lett., vol. 91, no. 23, p. 234 103, Dec. 2007.

[11] B. Kim, R. Candler, M. A. Hopcroft, M. Agarwal, W. T. Park, and T. W. Kenny, "Frequency stability of wafer-scale film encapsulated silicon based MEMS resonators," Sens. Actuators A, Phys., vol. 136, no. 1, pp. 125-131, May 2007. 